\title{
Simulation models predict that school-age children are responsible for most human-to-mosquito Plasmodium falciparum transmission in southern Malawi
}

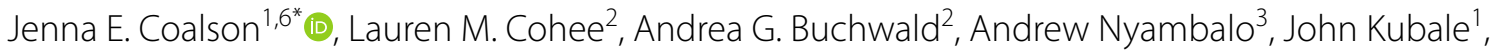
Karl B. Seydel ${ }^{4}$, Don Mathanga ${ }^{5}$, Terrie E. Taylor ${ }^{4}$, Miriam K. Laufer ${ }^{2}$ and Mark L. Wilson ${ }^{1 *}$

\begin{abstract}
Background: Malaria persists in some high-transmission areas despite extensive control efforts. Progress toward elimination may require effective targeting of specific human populations that act as key transmission reservoirs.

Methods: Parameterized using molecular-based Plasmodium falciparum infection data from cross-sectional community studies in southern Malawi, a simulation model was developed to predict the proportions of human-to-mosquito transmission arising from (a) children under 5 years old (U5s), (b) school-age children (SAC, 5-15 years), (c) young adults (16-30 years), and (d) adults $>30$ years. The model incorporates mosquito biting heterogeneity and differential infectivity (i.e. probability that a blood-fed mosquito develops oocysts) by age and gametocyte density.

Results: The model predicted that SAC were responsible for more than $60 \%$ of new mosquito infections in both dry and rainy seasons, even though they comprise only $30 \%$ of this southern Malawi population. Young adults were the second largest contributors, while U5s and adults over 30 were each responsible for $<10 \%$ of transmission. While the specific predicted values are sensitive to the relative infectiousness of SAC, this group remained the most important contributor to mosquito infections under all realistic estimates.
\end{abstract}

Conclusions: These results suggest that U5 children play a small role compared to SAC in maintaining P. falciparum transmission in southern Malawi. Models that assume biting homogeneity overestimate the importance of U5s. To reduce transmission, interventions will need to reach more SAC and young adults. This publicly available model can be used by others to estimate age-specific transmission contributions in epidemiologically similar sites with local parameter estimates of $P$. falciparum prevalence and bed net use.

Keywords: Malaria, Transmission reservoirs, School-age children, Simulation model, Plasmodium falciparum, Gametocytes

\footnotetext{
*Correspondence: jcoalson@gmail.com; wilsonml@umich.edu

${ }^{1}$ Department of Epidemiology, School of Public Health, University

of Michigan, M5507 SPH II, 1415 Washington Heights, Ann Arbor, Ml

48109, USA

Full list of author information is available at the end of the article
} 


\section{Background}

Although malaria control interventions have reduced parasite prevalence in some regions, ambitious efforts have caused little reduction in many settings with high endemicity [1-6]. Progress toward elimination requires understanding why current strategies fail. One potential explanation is that they do not adequately target sources of human-to-mosquito Plasmodium transmission. Defining the primary human reservoirs of transmission, or people who contribute most to infecting competent vectors, requires an understanding of infection patterns in human populations, variations in infectivity when bitten, and differential likelihoods of being bitten by female Anopheles.

Southern Malawi is one area where, despite high coverage of insecticide-treated nets (ITNs), the malaria burden remains high. Recent cross-sectional studies have found that 5-15-year old, school-age children (SAC) have the highest prevalence of Plasmodium falciparum infection [7], are more likely to carry gametocytes when parasitaemic [8], and are less likely to use bed nets [9] than children under five (U5s) and adults over 15 years. These findings suggest that SAC may be important human-tomosquito transmission reservoirs in that area, but their contribution has not been quantified.

Several skin- or membrane-feeding studies in other sites have calculated age-specific contributions of human infectious reservoir populations [10-18]. While they elucidated the difference between infected and infectious humans, most models did not consider whether mosquito feeding heterogeneity influences which people are key transmission reservoirs. Indeed, highly infectious people who are never bitten cannot contribute to transmission, while weakly infectious people who are frequently bitten might contribute considerably. Mosquito feeding frequency has been found to increase with the age of human hosts, possibly due in part to increasing body surface area [18-22]. Recently, Gonçalves et al. [18] matched mosquito blood meals to their human sources and found evidence of extreme heterogeneity in biting frequency by age. In the high-transmission setting of Burkina Faso, adults were bitten 20 times as often as U5s, and SAC were bitten 7 times as often as U5s, and, accordingly, the adult contribution to transmission was underestimated when considering only the proportion of infectious people by age [18]. Assuming blood-feeding homogeneity limits the estimation of true transmission contributions.

The present study aimed to estimate human, age-specific transmission of $P$. falciparum to mosquitoes, based on 3 years of surveillance data from southern Malawi, using a model that incorporated heterogeneity in mosquito exposure to different age-groups due to differential
ITN use and likelihood of being bitten. The hypothesis was that SAC are the primary contributors to transmission, particularly during the dry season. A secondary aim was to develop a simple modelling tool to enable researchers or policy-makers in other settings to evaluate relative transmission by different human groups using key surveillance data on parasite prevalence and ITN use.

\section{Methods}

\section{Ethics, consent, and permissions}

The human infection data used in this model were derived from repeated cross-sectional studies in southern Malawi. Informed consent was obtained from all participants or their guardians; assent was also obtained from 13 to 17 year old participants. The study protocol was approved by the independent Institutional Review Boards of the University of Malawi College of Medicine, the University of Maryland, Baltimore, and Michigan State University.

\section{Study setting}

Southern Malawi has perennially endemic malaria, with elevated incidence during the rainy season [3]. P. falciparum is predominant, though Plasmodium ovale and Plasmodium malariae infections also occur (typically as co-infections with P. falciparum) [23]. Cross-sectional data were gathered at the ends of the dry and rainy seasons from 2012 to 2015 from sites sampled in three ecologically distinct districts: urban highlands with relatively low transmission, rural highlands with moderate transmission, and rural lowlands with high transmission.

\section{Model and analysis}

A mathematical model was developed to estimate the proportion of new mosquito infections attributable to people in four age categories: (1) young children, < 5 years; (2) SAC, 5-15 years; (3) young adults, $>15-30$ years; and (4) adults, $>30$ years (Fig. 1). Their contributions were estimated statically for the ends of the dry and rainy seasons.

Estimating the number of new mosquito infections attributable to each age group, $M_{a}$, involved three components: (1) the number of people in each age group with P. falciparum infection, $n_{a}$, (2) the probability of humanto-mosquito transmission in a single blood meal from an infected person, or human infectivity, $\kappa$, and (3) the relative number of blood meals taken per human age group, $b_{a}$. Human-to-mosquito infectivity, $\mathrm{K}$, was considered dependent on gametocyte density $(d)$ in all iterations of the model output, with additional effects of age $(a)$ in some sensitivity analyses.

As a simplifying assumption, the population was divided into four categories of age and four categories of 


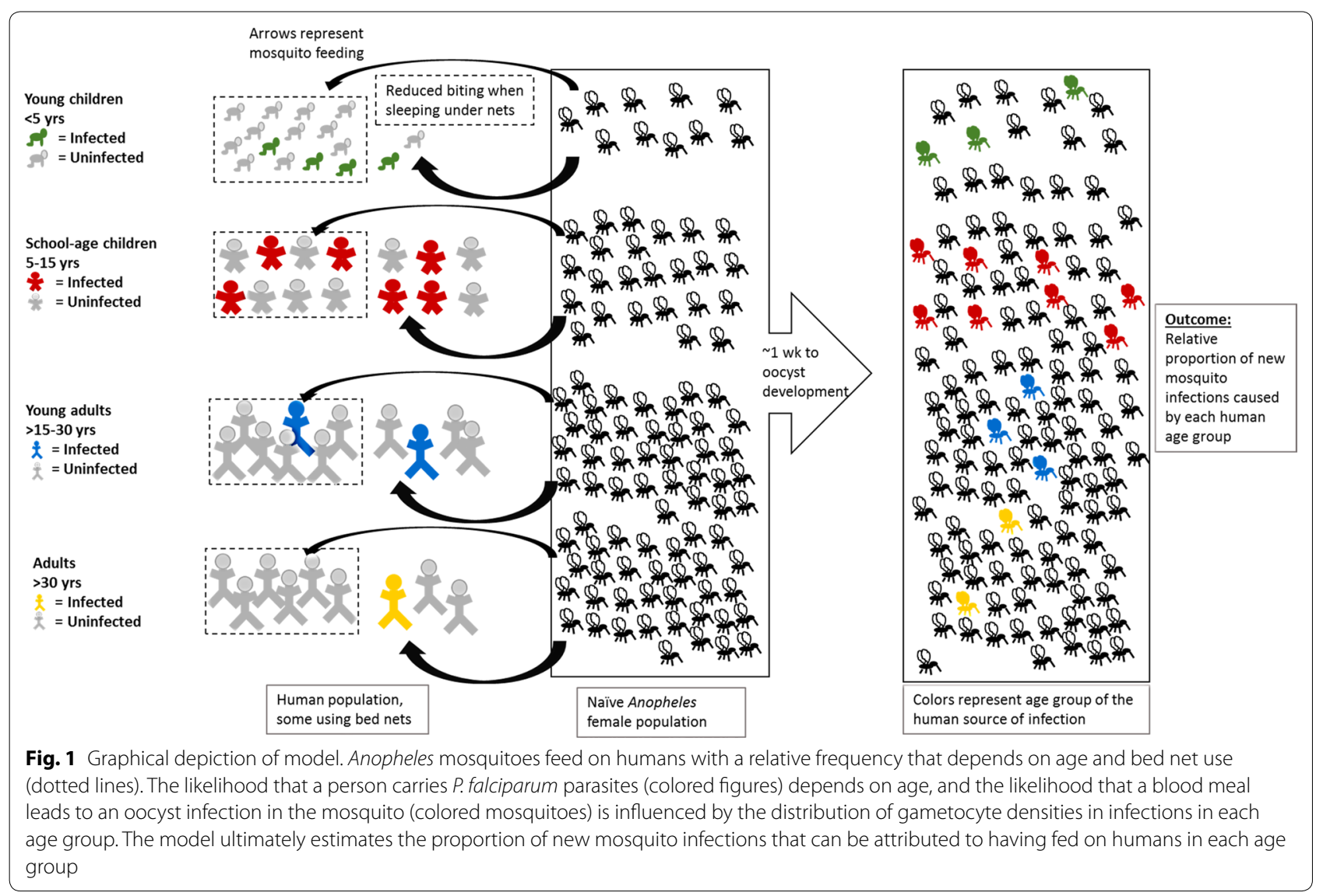

gametocyte density, for a total of 16 compartments. Densities were categorized as: (1) less than one gametocyte per $\mu \mathrm{L}$, (2) one to 200 gametocytes per $\mu \mathrm{L}$, (3) 201-400 gametocytes per $\mu \mathrm{L}$, and (4) more than 400 gametocytes per $\mu \mathrm{L}$ [24]. The number of people within each of the 16 age and gametocyte density categories, $n_{a, d}$, was calculated from the total number of people in the age group, $N_{a}$, based on census data, the prevalence of $P$. falciparum infection, $\alpha_{a}$, in age group $a$ estimated by quantitative polymerase chain reaction (qPCR), and the proportion of qPCR-positive infections in the age group in which the density of gametocytes is $d, g_{a, d}$, as:

$$
n_{a, d}=N_{a} \alpha_{a} g_{a, d}
$$

Differential prevalence of infection by age may be due to risk of becoming infected or of infection duration, the latter being influenced by acquired immune responses and variation in treatment-seeking. Since our model was static, and calculated reservoir contributions for certain times of the year based on measured prevalences, these prevalence parameters were used without disentangling the root causes.

Human-to-mosquito infectivity was assumed in all iterations of the model to increase with gametocyte density, $\kappa_{d}$ [24-26]. Differences in infectivity by age, $\kappa_{a}$, that are independent of the effect of gametocyte density are difficult to measure and poorly defined, but may be influenced by recent treatments, transmission-blocking immunity, and other unknown factors. Accordingly, estimates from the literature were used to establish the best estimates for baseline values and to estimate reasonable ranges for a sensitivity analysis.

The relative biting frequency by age, $b_{a}$, was calculated from: (1) estimated relative biting preference by age, $\delta_{a}$, as used by Stone et al. [21], (2) the prevalence of ITN use in each age group, $\sigma_{a}$, and (3) the relative reduction in biting frequency attributable to ITN use, $\gamma$, as:

$$
b_{a}=\delta_{a}\left(\sigma_{a} \gamma+\left(1-\sigma_{a}\right)\right)
$$

The number of new mosquito infections attributable to each age group, $M_{a}$ was then calculated as:

$$
M_{a}=\kappa_{a} b_{a} \sum_{d=1}^{4}\left(n_{a, d} k_{d}\right)
$$

As several parameters were calculated as relative estimates across age groups rather than as exact 
quantifications, the results for $M_{a}$ were used to calculate the proportion of new mosquito infections attributable to each age group, $F_{a}$ :

$$
F_{a}=\frac{M_{a}}{\sum_{a=1}^{4} M_{a}}
$$

Brief descriptions and sources of data for each parameter are provided in Table 1.

\section{Study design for primary data collection}

Reported ITN use, $\sigma_{a}$, and molecular data characterizing parasite prevalence, $\alpha_{a}$, by age for both seasons were obtained from cross-sectional studies carried out every 6 months from 2012 to 2014; data on gametocyte density distribution by age and season, $g_{a, d}$, were obtained from additional surveys that occurred in a subset of sites in the rainy and dry seasons of 2015 .

\section{Parasite prevalence and net use}

The design of the original six surveys has been previously reported [7, 9]; briefly, clusters of 30 households within ten enumeration areas were chosen by cluster-random sampling in each of the three study districts. All present and consenting members of these $\sim 900$ households were surveyed regarding malaria symptoms, treatments, and ownership/use of ITNs. Participants over 6 months of age had finger-prick blood sampled for qPCR. qPCR was run in singlicate with a limit of detection of 2.7 parasites per $\mu \mathrm{L}$; $P$. falciparum infection was considered to be present if the sample produced a positive curve for Pf lactate dehydrogenase (LDH) $[7,29]$. Molecular data on gametocyte density were not available for the samples taken from 2012 to 2014, so the distribution of gametocyte densities into the four density compartments by age category and season were predicted using molecular gametocyte data collected in 2015, as described in the next section.

\section{Gametocyte density distribution}

In 2015, four of the sites from the two rural districts were chosen for an extension study to schools. For each site, members of the original 30 households were included with an additional 50 households and cohorts of $\sim 100$ students in the nearest primary school. All participants provided finger-prick blood on filter paper for qPCR and preserved in RNAprotect ${ }^{\circledR}$ (Qiagen Inc., Valencia, CA) for quantitative reverse transcription PCR (qRT-PCR).

Gametocyte density distributions were measured among P. falciparum carriers from the 2015 household samples and the baseline school cohort samples. qPCR targeting LDH was run in duplicate to screen for infection. If either well was positive, qRT-PCR was performed

Table 1 Model parameter description and data sources

\begin{tabular}{|c|c|c|c|}
\hline Parameter & Description & Source & Value \\
\hline$n_{a, d}$ & $\begin{array}{l}\text { Number of humans of age group a that are P. falciparum-infected with } \\
\text { gametocytes at density } d \text { in Southern Malawi }\end{array}$ & Calculated, Eq. 1 & $n_{a, d}=N_{a} \alpha_{a} g_{a, d}$ \\
\hline$N_{a}$ & Number of people in age group a in Southern Malawi & 2008 Census [27] & \\
\hline$a_{a}$ & Prevalence of $P$. falciparum in age group $a$ & ICEMR-Malawi primary data & See Table 2 \\
\hline$g_{a, d}$ & Proportion of infections in age group a with gametocyte density $d$ & ICEMR-Malawi primary data & See Table 2 \\
\hline$K_{d}$ & $\begin{array}{l}\text { Percent of mosquitoes infected with oocysts after a blood meal from a } \\
\text { person with gametocytes at density } d\end{array}$ & Churcher et al. [24] & $\begin{array}{l}0.015 d=1(<1 / \mu \mathrm{L}) \\
0.04 d=2(1-200 / \mu \mathrm{L}) \\
0.10 d=3(>200-400 / \mu \mathrm{L}) \\
0.18 d=4(>400 / \mu \mathrm{L})\end{array}$ \\
\hline$\kappa_{a}$ & Relative infectivity per blood meal by age category a & $\begin{array}{l}\text { Ouédraogo et al. [17] } \\
\text { (including personal data } \\
\text { communication) }\end{array}$ & $\begin{array}{l}2.5 a=1(<5 \text { years }) \\
1.7 a=2(5-15 \text { years }) \\
1.0 \text { (ref) } a=3 \text { (> } 15-30 \text { years) } \\
0.6 a=4 \text { (>30 years) }\end{array}$ \\
\hline$b_{a}$ & Relative mosquito biting frequency by human age group a & Calculated, Eq. 2 & $b_{a}=\delta_{a}\left(\sigma_{a} \gamma+\left(1-\sigma_{a}\right)\right)$ \\
\hline$\delta_{a}$ & Vector's relative feeding preference for human age group a & Stone et al. [21] & $\begin{array}{l}1.0 \text { (ref) } a=1(<5 \text { years) } \\
3.0 a=2 \text { ( } 5-15 \text { years) } \\
4.0 a=3 \text { ( }>15-30 \text { years) } \\
4.0 a=4 \text { ( }>30 \text { years) }\end{array}$ \\
\hline$g_{a}$ & Proportion of people in age group a who use a bed net & ICEMR-Malawi primary data & See Table 2 \\
\hline Y & $\begin{array}{l}\text { Relative reduction in nightly biting frequency for bed net users compared } \\
\text { to non-users }\end{array}$ & Killeen et al. [28] & 0.40 \\
\hline$M_{a}$ & $\begin{array}{l}\text { Relative number of new mosquito oocyst infections attributable to human } \\
\text { age group a }\end{array}$ & Calculated, Eq. 3 & $M_{a}=\kappa_{a} b_{a} \sum_{d=1}^{4}\left(n_{a, d} k_{d}\right)$ \\
\hline$F_{a}$ & Fraction of all new mosquito infections attributable to human age group a & Calculated, Eq. 4 & $F_{a}=\frac{M_{a}}{\sum_{a=1}^{4} M_{a}}$ \\
\hline
\end{tabular}


to detect the mature gametocyte marker Pfs $25[25,30-$ 32]. Gametocyte density was quantified using a standard curve derived from cultured and diluted Pf.2004 TdT P. falciparum. Additional detail on gametocyte testing methods is provided as Additional file 1 .

For all PCR-positive infections, the age- and seasonspecific distributions of gametocyte densities were determined for the four gametocyte density categories defined above; some age- and season-specific compartments were empty. One individual was added to all compartments to smooth distribution estimates for the model.

The distribution of gametocyte density category by age and season in 2015 was assumed to apply to qPCR-positive infections from the 2012 to 2014 surveys, for which gametocyte data were not available. Total parasite density was significantly correlated with gametocyte density in 2015 ( $\mathrm{p}=0.03)$, and no significant difference was found in the association of age and total parasite density in the 2012-2014 dataset versus the 2015 dataset for either the rainy or dry season, supporting the assumption that the distribution of gametocyte densities by age and season were similar among PCR-positive cases in the two datasets.

\section{Secondary data for estimation of other parameter values}

The population distribution for Malawi's Southern Region was abstracted from the most recent census in 2008 [27]. Published journal articles guided estimation of human-to-mosquito infectivity based on age and gametocyte density, differential mosquito biting by age, and reduction in biting frequency associated with ITNs (Table 1).

Comparisons were made for predicted transmission contributions when assuming homogeneous biting, as in previous reports, to those that allowed for differential biting by age and ITN use. This model used the same estimates of relative biting as Stone et al. [21] based on differences in body surface area. The values are consistent with data from human landing catch studies, and may even underestimate the increase with age [18-22].
Investigators have researched human-to-mosquito infectivity for decades, but the determining factors remain controversial and poorly quantified (a summary of age-related infectivity data is provided as Additional file 2). It is widely accepted that infectivity increases with gametocyte density, though the exact shape of the association is still debated [24-26], so all model runs adjusted for a density-dependent infectivity parameter. Published studies generally indicate that infectiousness decreases with age, though methods vary widely, sample sizes tend to be small, few adjusted for gametocyte density, and findings are inconsistent [10-17, 33-43]. Data stratified by age and gametocyte status (i.e. microscopic versus submicroscopic) from a recent transmission study in Burkina Faso were used to estimate the baseline parameters for infectivity by age beyond the effects of gametocyte density in the present model, $\left(\kappa_{a}\right)$, as 2.5:1.7:1.0:0.6 for U5s, SAC, young adults, and adults $>30$, respectively (Table 1) [17]. Sensitivity analyses were carried out within a reasonable range of estimates of infectivity. A few studies reported slightly lower infectiousness for U5s and SAC than for adults [11,33,34], so the lower bound of $\kappa_{a}$ was set to 0.75 for both groups (relative to young adults). The upper bound for both was set to 4.0, as no published data supported a higher difference in infectivity by age after accounting for gametocyte density.

\section{Results}

Input data

SAC had the highest parasite prevalence in both seasons (Table 2). Using age distributions from the 2008 census, an estimated $12 \%$ of $P$. falciparum carriers at the end of the dry season were U5s, 50\% were SAC (5-15 years), $26 \%$ were young adults (16-30 years), and $11 \%$ were adults over 30 . The pattern was similar, but slightly more evenly distributed, at the end of the rainy season, when SAC comprised $44 \%$ of $P$. falciparum infections.

Most infections had 0 or $<1$ gametocyte $/ \mu \mathrm{L}$ detected. Higher densities ( $>200$ gametocytes $/ \mu \mathrm{L}$ ) were more common during the rainy season and in children than adults,

Table 2 Population characteristics by season and estimated distribution of human reservoirs of infection

\begin{tabular}{|c|c|c|c|c|c|c|c|}
\hline \multirow[t]{2}{*}{ Age group } & \multirow{2}{*}{$\begin{array}{l}\% \\
\text { of population } \\
\left(N_{a} / N\right)[27](\%)\end{array}$} & \multicolumn{3}{|l|}{ Dry season } & \multicolumn{3}{|l|}{ Rainy season } \\
\hline & & $\begin{array}{l}\text { P. falciparum } \\
\text { prevalence (qPCR), } \\
a_{a}(\%)\end{array}$ & $\begin{array}{l}\text { ITN } \\
\text { usage, } \\
\sigma_{a}(\%)\end{array}$ & $\begin{array}{l}\% \text { of } P . \\
\text { falciparum } \\
\text { infections }^{\mathrm{a}}(\%)\end{array}$ & $\begin{array}{l}P . \text { falciparum } \\
\text { prevalence (qPCR), } \\
a_{a}(\%)\end{array}$ & $\begin{array}{l}\text { ITN } \\
\text { usage, } \\
\sigma_{a}(\%)\end{array}$ & $\begin{array}{l}\% \text { of } P \text {. falciparum } \\
\text { infections }^{\mathrm{a}}(\%)\end{array}$ \\
\hline Children ( $<5$ years) & 18.2 & 7.7 & 53.4 & 12.4 & 13.6 & 70.1 & 13.8 \\
\hline SAC (5-15 years) & 30.0 & 18.9 & 33.6 & 50.2 & 26.6 & 49.0 & 44.3 \\
\hline $\begin{array}{l}\text { Young adults } \\
\text { (16-30 years) }\end{array}$ & 27.2 & 10.8 & 59.7 & 26.0 & 16.3 & 65.3 & 24.6 \\
\hline Adults (> 30 years) & 24.7 & 5.2 & 56.4 & 11.3 & 12.7 & 63.0 & 17.4 \\
\hline
\end{tabular}

${ }^{a}$ Estimated percent of parasite carriers that are from each age group, $\left(N_{a} / N\right)^{*} a_{a}$ 
but comprised a small proportion of infections in both seasons. The predicted distributions are displayed with the average qPCR-based prevalences by age and season in Fig. 2.

\section{Estimates of transmission contribution and sensitivity to biting homogeneity assumptions}

Figure 3 displays the predicted contributions to transmission by age under three assumptions: (1) mosquito feeding was completely homogeneous by age, as in many prior infectivity studies, (2) ITNs reduced feeding on people using them, but mosquitoes were otherwise equally likely to bite people regardless of age, and (3) ITNs reduced feeding and the biting likelihood differed by age. Notably, there was very little seasonal difference in the age-specific contributions to new mosquito infections under any condition.

Despite comprising only 30\% of the population, all three models predicted that SAC caused the majority ( $>50 \%$ ) of human-to-mosquito transmission of P. falciparum in southern Malawi in both seasons (Fig. 3). Relaxing the biting homogeneity assumption to account for heterogeneous ITN use and likelihood of biting based on body size increased SAC's predicted transmission contribution to more than $60 \%$. Adults over 30 , who comprised $\sim 25 \%$ of the population, were estimated to contribute little $(<10 \%)$ to new mosquito infections in either season under all heterogeneity assumptions.

Incorporating differential ITN use had less impact than assuming homogeneous likelihood of biting based on age/body size. Assuming homogeneous biting overestimated the contribution of U5s, who have small body surface area and high ITN use, and underestimated the contributions of SAC and young adults. Models that assumed homogeneous biting by age roughly tripled the predicted transmission contribution of U5s (who comprised $18.2 \%$ of the population), from 9.4 to $25.2 \%$ during the dry season, and 8.1 to $22.4 \%$ during the rainy season. Incorporating biting heterogeneity reduced their predicted contributions to $<10 \%$ and made young adults, who comprised $27.2 \%$ of the population, the second largest transmission contributors at 21.6 and $22.1 \%$.

\section{Sensitivity analyses for human infectivity to mosquitoes by age}

Given the uncertainty in the parameter value for infectivity by age, 4356 simulations were run per season with $\kappa_{<5}$ and $\kappa_{S A C}$ ranging from 0.75 to 4.0, incorporating mosquito biting heterogeneity and gametocyte density-dependent effects (Fig. 4). SAC were the largest contributors to transmission in southern Malawi under all age-dependent infectivity simulations, even for the most extreme conditions in which other age groups' contributions were maximized (Fig. 5). SAC were responsible for $>50 \%$ of transmission if they were at least $20 \%$ more infectious than young adults during the dry season and $25 \%$ more infectious than young adults during the rainy season, regardless of the relative infectivity of U5s. Young adults (16-30 years) were the second largest contributors to new mosquito infections under all potential infectivity scenarios for both seasons, although in most scenarios they were responsible for a smaller proportion of new transmission than their $27.2 \%$ share of the population. Adults over 30 , who

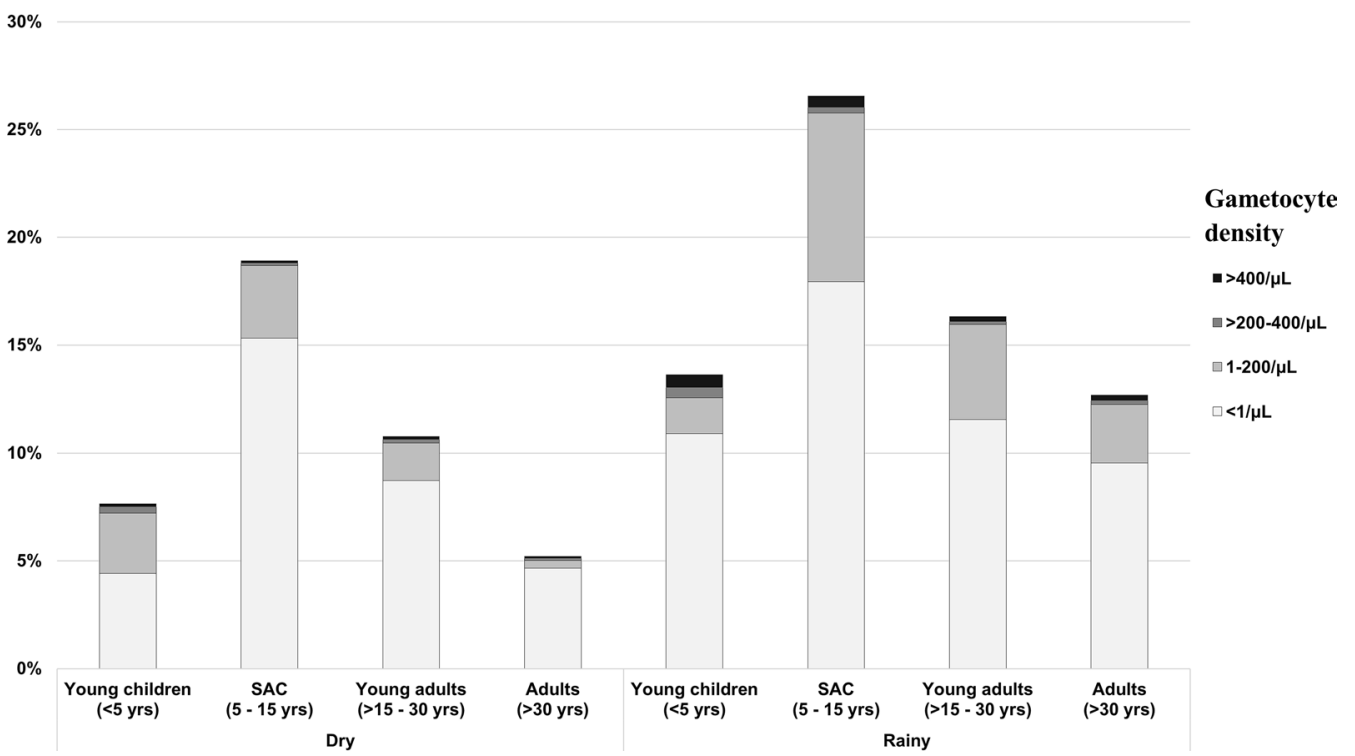

Fig. 2 qPCR-based prevalence of $P$. falciparum infection, stratified by qRT-PCR-predicted gametocyte density 


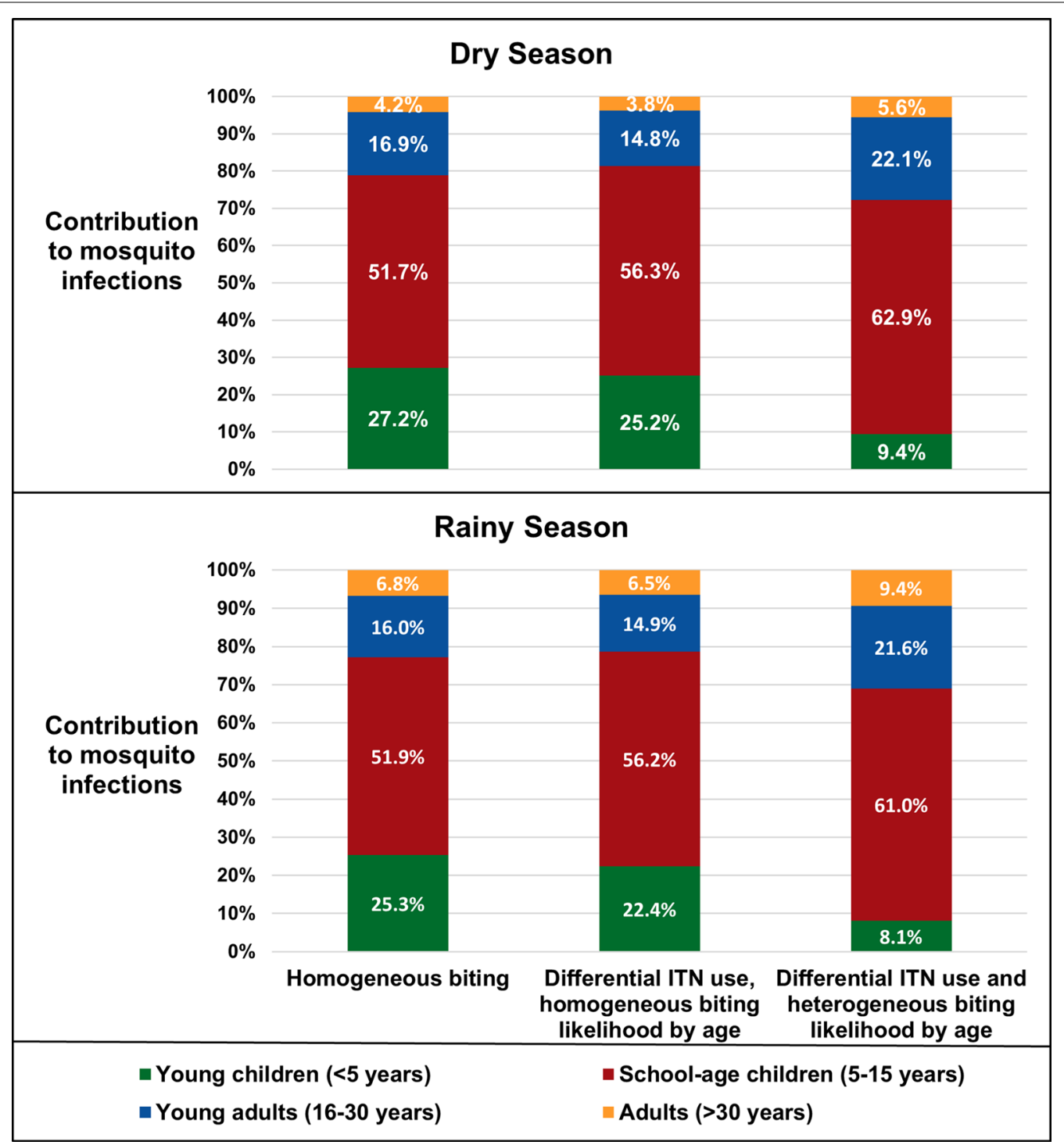

Fig. 3 Predicted transmission contributions under different assumptions about mosquito feeding frequency by human age

comprise $24.7 \%$ of the population, were responsible for $<10 \%$ of transmission in all dry season simulations and most rainy season simulations (Figs. 4, 5).

On the other hand, the predicted transmission contribution of U5s was sensitive to both their infectivity and the infectivity of SAC. Children under five comprise $18.2 \%$ of the population, but could only have contributed $\geq 18.2 \%$ of new mosquito infections if they were highly infectious relative to all other age groups, particularly during the rainy season. During the rainy season, even in the most extreme simulation, U5s would only have contributed a maximum of $18.3 \%$ of transmissions (Fig. 5). In the majority of simulated conditions, they were responsible for $<10 \%$ of transmission.
In all simulations, they contributed less to transmission than SAC and young adults.

\section{Discussion}

Using recent molecular parasite data and reported ITN use in southern Malawi, this model estimated that SAC were responsible for $>60 \%$ of $P$. falciparum transmission to mosquitoes during both the dry and rainy seasons. The model estimated that young adults (16-30 years) were the second largest contributors to transmission; U5 children and adults over 30 years were each predicted to have caused $<10 \%$ of mosquito infections in most simulations. The proportion of mosquito infections attributable to each human age group did not differ considerably by 


\begin{tabular}{|c|c|c|c|c|c|c|c|c|c|}
\hline \multirow[b]{2}{*}{ Age group } & \multirow{2}{*}{$\begin{array}{c}\% \text { of total } \\
\text { population, } \\
N_{a} / N \\
\end{array}$} & \multirow{2}{*}{$\begin{array}{l}\text { Relative biting } \\
\text { attractiveness, } \\
\delta\end{array}$} & \multirow{2}{*}{$\begin{array}{c}\text { Relative infectivity } \\
\text { to mosquito by age, } \\
\kappa_{a}\end{array}$} & \multicolumn{3}{|c|}{$\begin{array}{l}\text { Distribution of predicted contribution to } \\
\text { transmission, dry season }\end{array}$} & \multicolumn{3}{|c|}{$\begin{array}{l}\text { Distribution of predicted contribution to } \\
\text { transmission, rainy season }\end{array}$} \\
\hline & & & & Median & Range & IQR & Median & Range & IQR \\
\hline Young children ( $<5$ yrs) & $18.2 \%$ & 1 & $0.75-4.0$ & $7.16 \%$ & $1.58-21.31 \%$ & $4.80-9.90 \%$ & $6.22 \%$ & $1.38-18.33 \%$ & $4.15-8.59 \%$ \\
\hline SAC (5-15yrs) & $30.0 \%$ & 3 & $0.75-4.0$ & $70.60 \%$ & $39.40-82.92 \%$ & $61.05-76.39 \%$ & $68.80 \%$ & $37.99-81.13 \%$ & $59.00-74.82 \%$ \\
\hline Young adults (16-30 yrs) & $27.2 \%$ & 4 & 1.0 (ref) & $17.71 \%$ & $11.56-37.85 \%$ & $14.22-23.46 \%$ & $17.42 \%$ & $11.51-35.78 \%$ & $14.06-22.88 \%$ \\
\hline Adults (>30 yrs) & $24.7 \%$ & 4 & 0.6 & $4.53 \%$ & $2.96-9.68 \%$ & $3.64-6.00 \%$ & $7.57 \%$ & $5.00-15.54 \%$ & $6.11-9.94 \%$ \\
\hline
\end{tabular}
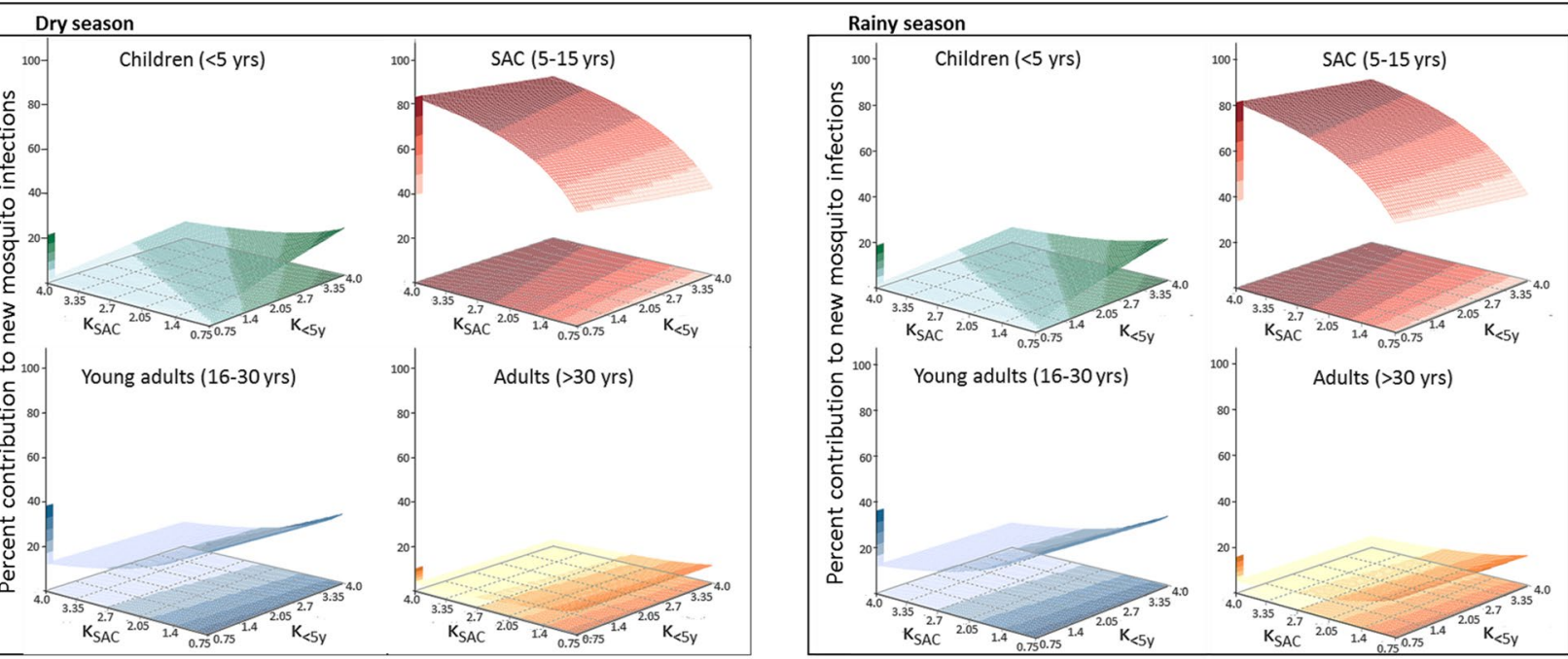

Abbreviations: IQR = Interquartile Range, $S A C=$ School-age children

Fig. 4 Predicted transmission contributions in a sensitivity analysis varying human-to-mosquito infectivity $\left(k_{a}\right)$ by age. Infectivity to mosquitoes may vary with human age in relation to differences in recent antimalarial treatment, treatment-blocking immunity, duration of infection, multiplicity of infection, or other unknown factors, all of which could differ with age, even after accounting for gametocyte presence and density. Thus a sensitivity analysis was performed for transmission contribution when varying infectivity by age with outside bounds informed by the scientific literature on membrane feeding experiments that accounted for gametocyte status. Infectivity of children under 5 years and school-age children were varied relative to the infectivity of young adults (16-30 years)

season. Lacking human-to-mosquito infectivity data from skin or membrane feeding, sensitivity analyses were performed under a reasonable range of estimates of relative infectivity by age after accounting for gametocyte density distributions. If U5s are far more infectious to mosquitoes than all other age groups at a given gametocyte density, their estimated transmission contribution approached $20 \%$, but SAC and young adults remained the largest and second largest contributors in these simulations. These findings may help explain $P$. falciparum's persistence in the area despite considerable malaria control efforts in the past decade which targeted groups at high risk of disease-pregnant women and children under five.

Predictions about the transmission reservoir are sensitive to heterogeneity in mosquito biting, particularly in evaluating the contribution of U5s. Several prior lab studies of infectivity have made simple estimates of agespecific transmission contributions; however, those reports assumed homogeneous mosquito feeding [1017]. The model presented here, like those of Stone et al. [21] and Gonçalves et al. [18], demonstrated that such a homogeneity assumption considerably overestimates the contribution of U5s and underestimates that of older age groups. In their mosquito blood meal source matching study, Gonçalves et al. [18] found that the degree of heterogeneity in mosquito biting by human age was different for their high and moderate transmission sites. The biting frequency ratio in the high transmission site was even more extreme than that used by Stone et al. and the model presented here, at 1:7:20 for U5s compared to SAC and adults 16 years and older, but the increase in biting frequency with age was moderate $(1: 2.3: 2.1)$ and did not achieve statistical significance in the low/moderate intensity transmission site in Kenya. Given the sensitivity of transmission dynamics to this parameter, future research should better quantify heterogeneity in human exposure to feeding mosquitoes to more accurately identify key $P$. falciparum transmission reservoirs, and assess the factors which drive variation in this age-specific biting heterogeneity in different geographic settings.

The results presented here using recent empirical data from southern Malawi predict high transmission contribution from SAC, and are consistent with those of Stone et al. [21] which were based on generic sub-Saharan African estimates of parasite prevalence and population distribution. This suggests that the findings may be generalizable 


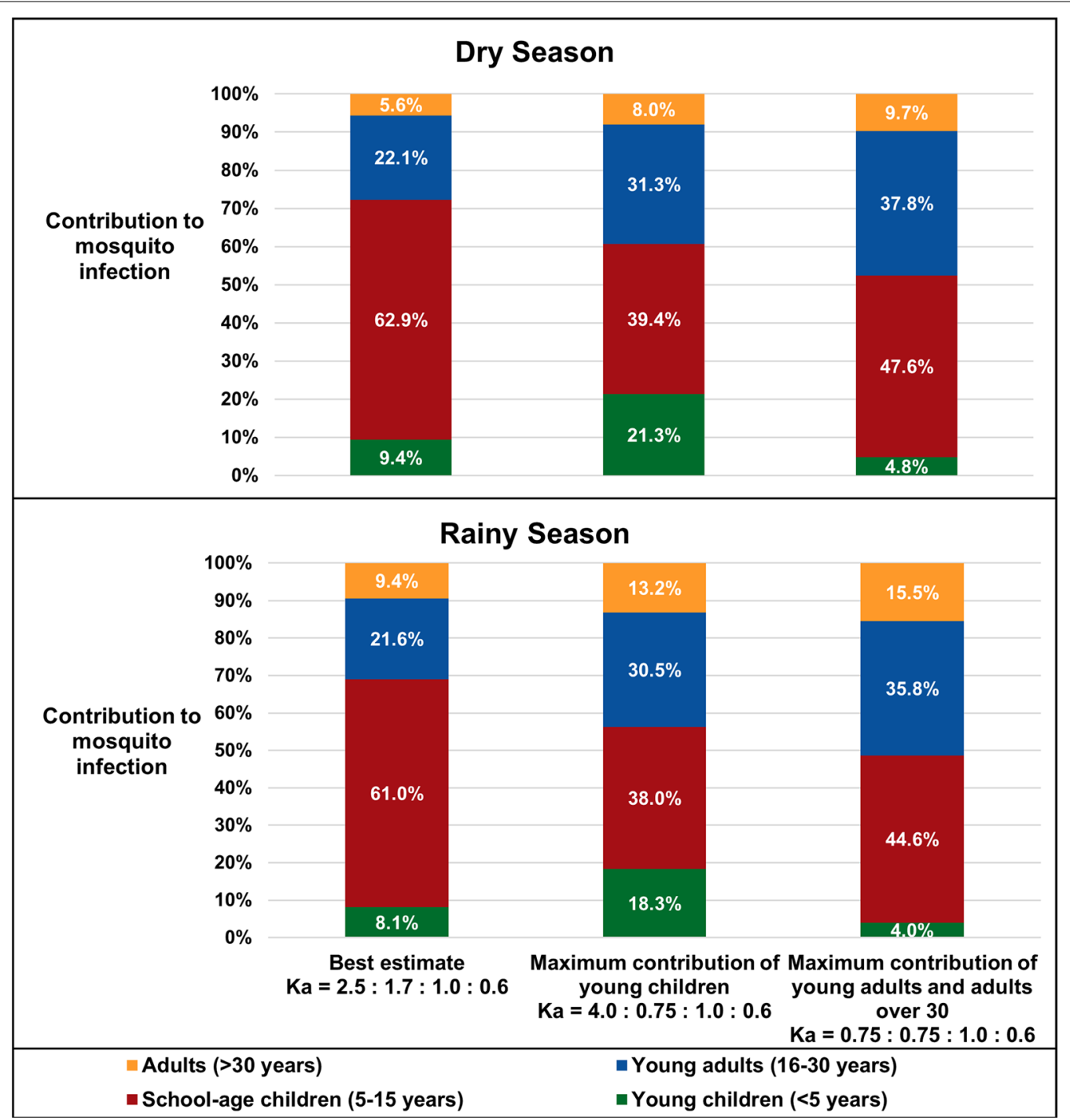

Fig. 5 Extreme possibilities of reservoir contributions from sensitivity analysis of human-to-mosquito infectivity $\left(k_{a}\right)$. Examples of extreme ends of the sensitivity analysis varying human-to-mosquito infectivity, as described in "Methods" and in Fig. 4

to other sub-Saharan African settings with high P. falciparum endemicity. A worksheet of the model calculations that other researchers can utilize in their specific contexts with, at a minimum, age-specific data on (1) population distribution; (2) estimated PCR prevalence; and (3) frequency of ITN use is provided as Additional file 3. Results should be interpreted with caution in settings with different malaria epidemiology, particularly those near elimination or where non-falciparum malaria is common.

A potential limitation of the study was the use of four compartments for gametocyte density, and the subsequent need to average the density-dependent transmission probability within each compartment. The estimation of parameters for each compartment based on Churcher et al. [24] resulted in a lower relative likelihood of transmission for the highest density categories compared to that applied in some other models [26]; however, the very small proportion of high density gametocyte carriers in this community-based population makes the model relatively insensitive to changes in the value selected for that compartment. This limitation would be critical, however, if the few high density gametocyte carriers are disproportionately likely to be fed upon by mosquitoes, essentially driving transmission as 'superspreaders'. As seen in this model, transmission patterns may be highly sensitive to heterogeneity in blood feeding. More research matching blood meals to specific human sources is needed to understand mosquito feeding patterns in real-world settings. If it is discovered that a select few superspreaders are responsible for the majority of 
Plasmodium transmission, this would have significant limitations for targeting interventions aimed at control and/or elimination.

Further, the model is static and depends on measured prevalence data, without regard for the causes of differential prevalence of infection by human age. While this should not limit inferences about transmission contributions for the time-period studied, design of effective interventions will require better understanding of whether differences in infection prevalence are a result of differences in risk of infection, natural infection clearance time, frequency of anti-malarial treatment, or some combination of these.

\section{Conclusions}

This model predicted that SAC and young adults were responsible for $>80 \%$ of all new mosquito infections in southern Malawi, with SAC contributing $\sim 60 \%$ overall despite comprising only $30 \%$ of the population. A critical implication is that malaria elimination is unlikely in persistent settings like southern Malawi unless researchers and policy-makers develop interventions that can successfully reduce transmission from these groups, who are often asymptomatic and submicroscopically infected. More sensitive rapid detection methods and development of safe/effective drugs for transmission reduction are therefore fundamental to further progress toward malaria eradication.

\section{Additional files}

Additional file 1. Additional detail on gametocyte testing methods. Text providing further detail on the molecular testing methods used for the detection of gametocytes.

Additional file 2. Summary of age-specific infectiousness data from membrane- and skin-feeding studies. Table providing abstracted data from a literature review of membrane- and skin-feeding studies, especially those that provided data on differential infectivity by age and any calculations of human contributions to the infectious or transmission reservoirs.

Additional file 3. Tool for site-specific model calculations. A spreadsheet that enables researchers and policy-makers to input data from their site into the model and estimate transmission contributions by age. Caution is recommended for the interpretation of this output in settings with malaria epidemiology that is very different than that of southern Malawi.

Additional file 4. Dry season model equations. Files for use with Berkeley Madonna modelling software that include model equations and dry season parameter estimates from this paper.

Additional file 5. Rainy season model equations. Files for use with Berkeley Madonna modelling software that include model equations and rainy season parameter estimates from this paper.

\section{Abbreviations}

ICEMR: International Center of Excellence for Malaria Research; ITN: insecticidetreated net; $\mathrm{LDH}$ : lactate dehydrogenase; GPCR: quantitative polymerase chain reaction; qRT-PCR: quantitative reverse transcription polymerase chain reaction; SAC: school-age children; U5s: children under 5 years of age.

\section{Authors' contributions}

JEC performed molecular testing, assisted with data and sample collection, completed data cleaning, created the statistical models, performed all model analyses, and drafted the manuscript. LMC assisted with data and sample collection and management, contributed to the design and conduct of the cross-sectional study, aided with conceptual design of the simulation models, and provided critical review of the manuscript. AGB assisted with conceptual design of the model, estimation of model parameters, and provided critical review of the manuscript. AN performed molecular testing and provided critical review of the manuscript. JK performed literature reviews, assisted with estimation of model parameters, and provided critical review of the manuscript. KBS managed data processing and molecular testing and provided critical review of the manuscript. DM and TET both contributed to the design and conduct of the cross-sectional study and provided critical review of the manuscript. MKL contributed to the design and conduct of the cross-sectional study, aided with conceptual design of the model, and provided critical review of the manuscript. MLW aided with conceptual design of the model, estimation of model parameters and interpretation of results, and provided critical review of the manuscript. All authors read and approved the final manuscript.

\section{Author details}

${ }^{1}$ Department of Epidemiology, School of Public Health, University of Michigan, M5507 SPH II, 1415 Washington Heights, Ann Arbor, MI 48109, USA. ${ }^{2}$ Division of Malaria Research, Institute of Global Health, University of Maryland School of Medicine, Baltimore, MD, USA. ${ }^{3}$ Blantyre Malaria Project, University of Malawi College of Medicine, Blantyre, Malawi. ${ }^{4}$ College of Osteopathic Medicine, Michigan State University, East Lansing, USA. ${ }^{5}$ Malaria Alert Centre, University of Malawi College of Medicine, Blantyre, Malawi. ${ }^{6}$ Present Address: Center for Insect Science and Department of Epidemiology and Biostatistics, University of Arizona, Tucson, AZ, USA.

\section{Acknowledgements}

Our sincere thanks to the field team, staff coordinators, laboratory technicians, and study participants from the cross-sectional studies, without whom this analysis would not be possible. We would also like to thank Dr. Matthias Marti for provision of the Pf.2004 TdT P. falciparum strain used for gametocyte standard curves, and Drs. Andre Lin Ouedraogo, Edward A. Wenger, and Philip A. Eckhoff from IDM for providing detailed infectivity data related to their 2016 publication to enable additional stratification that was not included in the original published analysis [17].

Competing interests

The authors declare that they have no competing interests.

\section{Availability of data and materials}

The models supporting the conclusions of this article are included as Additional files 4 and 5 for Berkeley Madonna modelling software, and can be provided in other formats upon request. The raw data used to estimate parameters for this model are part of an ongoing study. All data will be deidentified and made available after the study's conclusion.

\section{Consent for publication}

Not applicable.

\section{Ethics approval and consent to participate}

The study which provided raw data for parameter estimation relevant to southern Malawi was approved by the independent Institutional Review Boards of the University of Malawi College of Medicine, the University of Maryland, Baltimore, and Michigan State University. Informed consent was obtained for all individuals included in the study.

\section{Funding}

This work was supported by the National Institute of Allergy and Infectious Diseases (NIAID) [U19AI089683 and R21Al107421] and the National Institute of General Medical Sciences (NIGMS) [K12 GM000708] at the National Institutes of Health (NIH).

\section{Publisher's Note}

Springer Nature remains neutral with regard to jurisdictional claims in published maps and institutional affiliations. 
Received: 30 November 2017 Accepted: 24 March 2018

Published online: 03 April 2018

\section{References}

1. Snow RW. Global malaria eradication and the importance of Plasmodium falciparum epidemiology in Africa. BMC Med. 2015;13:23.

2. Wilson ML, Walker ED, Mzilahowa T, Mathanga DP, Taylor TE. Malaria elimination in Malawi: research needs in highly endemic, poverty-stricken contexts. Acta Trop. 2012;121:218-26.

3. Mathanga DP, Walker ED, Wilson ML, Ali D, Taylor TE, Laufer MK. Malaria control in Malawi: current status and directions for the future. Acta Trop. 2012;121:212-7

4. Noor AM, Kinyoki DK, Mundia CW, Kabaria CW, Mutua JW, Alegana VA, et al. The changing risk of Plasmodium falciparum malaria infection in Africa: 2000-10: a spatial and temporal analysis of transmission intensity. Lancet. 2014:383:1739-47.

5. WHO. World malaria report 2015. Geneva: World Health Organization 2015

6. Murray CJL, Ortblad KF, Guinovart C, Lim SS, WolockTM, Roberts DA, et al. Global, regional, and national incidence and mortality for HIV, tuberculosis, and malaria during 1990-2013: a systematic analysis for the Global Burden of Disease Study 2013. Lancet. 2014:384:1005-70.

7. Walldorf JA, Cohee LM, Coalson JE, Bauleni A, Nkanaunena K, KapitoTembo A, et al. School-age children are a reservoir of malaria infection in Malawi. PLoS ONE. 2015;10:e0134061.

8. Coalson JE, Walldorf JA, Cohee LM, Ismail MD, Mathanga D, Joice R, et al. High prevalence of Plasmodium falciparum gametocyte infections in school-age children using sensitive molecular detection: patterns and predictors of risk from a cross-sectional study in southern Malawi. Malar J. 2016;15:527.

9. Buchwald A, Walldorf J, Cohee L, Coalson J, Chimbiya N, Bauleni A, et al. Bed net use among school-aged children after a universal bed net campaign in Malawi. Malar J. 2016:15:127.

10. Muirhead-Thomson RC. The malarial infectivity of an African village population to mosquitoes (Anopheles gambiae): a random xenodiagnostic survey. Am J Trop Med Hyg. 1957;6:971-9.

11. Gamage-Mendis AC, Rajakaruna J, Carter R, Mendis KN. Infectious reservoir of Plasmodium vivax and Plasmodium falciparum malaria in an endemic region of Sri Lanka. Am J Trop Med Hyg. 1991;45:479-87.

12. Githeko AK, Brandling-Bennett AD, Beier M, Atieli F, Owaga M, Collins FH. The reservoir of Plasmodium falciparum malaria in a holoendemic area of western Kenya. Trans R Soc Trop Med Hyg. 1992:86:355-8.

13. Boudin C, Olivier M, Molez J-F, Chiron J-P, Ambroise-Thomas P. High human malarial infectivity to laboratory-bred Anopheles gambiae in a village in Burkina Faso. Am J Trop Med Hyg. 1993;48:700-6.

14. Drakeley C, Akim N, Sauerwein R, Greenwood B, Targett G. Estimates of the infectious reservoir of Plasmodium falciparum malaria in The Gambia and in Tanzania. Trans R Soc Trop Med Hyg. 2000;94:472-6.

15. Bonnet S, Gouagna LC, Paul RE, Safeukui I, Meunier JY, Boudin C. Estimation of malaria transmission from humans to mosquitoes in two neighbouring villages in south Cameroon: evaluation and comparison of several indices. Trans R Soc Trop Med Hyg. 2003;97:53-9.

16. Gaye A, Bousema T, Libasse $G$, Ndiath $M O$, Konaté L, Jawara M, et al. Infectiousness of the human population to Anopheles arabiensis by direct skin feeding in an area hypoendemic for malaria in Senegal. Am J Trop Med Hyg. 2015;92:648-52.

17. Ouédraogo AL, Gonçalves BP, Gnémé A, Wenger EA, Guelbeogo MW Ouédraogo A, et al. Dynamics of the human infectious reservoir for malaria determined by mosquito feeding assays and ultrasensitive malaria diagnosis in Burkina Faso. J Infect Dis. 2016;213:90-9.

18. Gonçalves BP, Kapulu MC, Sawa P, Guelbéogo WM, Tiono AB, Grignard $L$, et al. Examining the human infectious reservoir for Plasmodium falciparum malaria in areas of differing transmission intensity. Nat Commun. 2017;8:1133.

19. Carnevale P, Frézil J, Bosseno M, Le Pont F, Lancien J. Etude de l'aggressivité d'Anopheles gambiae A en fonction de l'âge et du sexe des subjets humains. Bull World Health Organ. 1978;56:147-54.
20. Port GR, Boreham PFL, Bryan JH. The relationship of host size to feeding by mosquitoes of the Anopheles gambiae Giles complex (Diptera: Culicidae). Bull Entomol Res. 1980:70:133-44.

21. Stone W, Gonçalves BP, Bousema T, Drakeley C. Assessing the infectious reservoir of falciparum malaria: past and future. Trends Parasitol. 2015;31:287-96.

22. Muirhead-Thomson RC. The distribution of anopheline mosquito bites among different age groups: a new factor in malaria epidemiology. BMJ. 1951;1:1114-7.

23. Bruce MC, Macheso A, Kelly-Hope LA, Nkhoma S, McConnachie A, Molyneux ME. Effect of transmission setting and mixed species infections on clinical measures of malaria in Malawi. PLoS ONE. 2008;3:e2775.

24. Churcher TS, Bousema T, Walker M, Drakeley C, Schneider P, Ouédraogo $A L$, et al. Predicting mosquito infection from Plasmodium falciparum gametocyte density and estimating the reservoir of infection. Elife. 2013;2:e00626.

25. Bousema T, Drakeley C. Epidemiology and infectivity of Plasmodium falciparum and Plasmodium vivax gametocytes in relation to malaria control and elimination. Clin Microbiol Rev. 2011;24:377-410.

26. Johnston GL, Smith DL, Fidock DA. Malaria's missing number: calculating the human component of $\mathrm{R}_{0}$ by a within-host mechanistic model of Plasmodium falciparum infection and transmission. PLoS Comput Biol. 2013;9:e1003025

27. National Statistical Office. 2008 population and housing census: preliminary report. 2008 (September).

28. Killeen GF, Smith TA, Ferguson HM, Mshinda H, Abdulla S, Lengeler C, et al. Preventing childhood malaria in Africa by protecting adults from mosquitoes with insecticide-treated nets. PLoS Med. 2007:4:e229.

29. Rantala A-M, Taylor SM, Trottman PA, Luntamo M, Mbewe B, Maleta K, et al. Comparison of real-time PCR and microscopy for malaria parasite detection in Malawian pregnant women. Malar J. 2010;9:269.

30. Alano P. Molecular approaches to monitor parasite genetic complexity in the transmission of Plasmodium falciparum malaria. Parassitologia. 2005:47:199-203.

31. Mwingira F, Genton B, Kabanywanyi ANM, Felger I. Comparison of detection methods to estimate asexual Plasmodium falciparum parasite prevalence and gametocyte carriage in a community survey in Tanzania. Malar J. 2014:13:433.

32. Wampfler R, Mwingira F, Javati S, Robinson L, Betuela I, Siba P, et al. Strategies for detection of Plasmodium species gametocytes. PLoS ONE. 2013;8:e76316.

33. Drakeley CJ, Secka I, Correa S, Greenwood BM, Targett GAT. Host haematological factors influencing the transmission of Plasmodium falciparum gametocytes to Anopheles gambiae s.s. mosquitoes. Trop Med Int Health. 1999:4:131-8.

34. Tchuinkam T, Mulder B, Dechering K, Stoffels H, Verhave JP, Cot M, et al. Experimental infections of Anopheles gambiae with Plasmodium falciparum of naturally infected gametocyte carriers in Cameroon: factors influencing the infectivity to mosquitoes. Trop Med Parasitol. 1993:44:271-6.

35. Toure YT, Doumbo O, Toure A, Bagayoko M, Diallo M, Dolo A, et al. Gametocyte infectivity by direct mosquito feeds in an area of seasonal malaria transmission: implications for Bancoumana, Mali as a transmission-blocking vaccine site. Am J Trop Med Hyg. 1998;59:481-6.

36. Draper CC. Observations on the infectiousness of gametocytes in hyperendemic malaria. Trans R Soc Trop Med Hyg. 1953;47:160-5.

37. Graves PM, Burkot TR, Carter R, Cattani JA, Lagog M, Parker J, et al. Measurement of malarial infectivity of human populations to mosquitoes in the Madang area, Papua New Guinea. Parasitology. 1988;96(Pt 2):251-63.

38. Haji H, Smith T, Charlwood JD, Meuwissen JH. Absence of relationships between selected human factors and natural infectivity of Plasmodium falciparum to mosquitoes in an area of high transmission. Parasitology. 1996:113:425-31.

39. Coleman RE, Kumpitak C, Ponlawat A, Phunkitchar V, Rachapaew N, Zollner G. Infectivity of asymptomatic Plasmodium-infected human populations to Anopheles dirus mosquitoes in Western Thailand. J Med Entomol. 2004:41:201-8.

40. Muirhead-Thomson R. Low gametocyte thresholds of infection of Anopheles with Plasmodium falciparum: a significant factor in malaria epidemiology. BMJ. 1954;1:68-70

41. Ouédraogo AL, Bousema T, Schneider P, de Vlas SJ, Ilboudo-Sanogo E, Cuzin-Ouattara N, et al. Substantial contribution of submicroscopical 
Plasmodium falciparum gametocyte carriage to the infectious reservoir in an area of seasonal transmission. PLoS ONE. 2009;4:e8410.

42. Schneider P, Bousema JT, Gouagna LC, Otieno S, Van de Vegte-Bolmer M, Omar SA, et al. Submicroscopic Plasmodium falciparum gametocyte densities frequently result in mosquito infection. Am J Trop Med Hyg. 2007;76:470-4.
43. Mulder B, Tchuinkam T, Dechering K, Verhave JP, Carnevale P, Meuwissen JHET, et al. Malaria transmission-blocking activity in experimental infections of Anopheles gambiae from naturally infected Plasmodium falciparum gametocyte carriers. Trans R Soc Trop Med Hyg. 1994;88:121-5.

\section{Submit your next manuscript to BioMed Central and we will help you at every step:}

- We accept pre-submission inquiries

- Our selector tool helps you to find the most relevant journal

- We provide round the clock customer support

- Convenient online submission

- Thorough peer review

- Inclusion in PubMed and all major indexing services

- Maximum visibility for your research

Submit your manuscript at

www.biomedcentral.com/submit 